\title{
Fuzzy Control and its Applications in QCM Sensor System
}

\author{
CHEN Haixia \\ Tonghua Teachers College, China \\ thtchx@yeah.net
}

Keywords: Neural Networks, Pattern Recognition, Numerical Analysis.

\begin{abstract}
Research in the field of neural networks has made significant progress, that progress has attracted a lot of attention and support of the people more money on. Now more and more academic and commercial research is carried out on neural networks, such as chip-based neural networks are developed and applied, and produces a number of complex issues, and treats these problems solved. Obviously, now just a transition period of neural networks. Neural network results from the ability of complex data extraction can be used to extract or detect those patterns for humans or other computer technology is too complex hard to notice a trend. A class of design problems $\mathrm{H} \infty$ fuzzy neural network controller. By using Lyapunov-Krasovskii functional theory and judgment theorem to derive a stable process introduces several additional matrices, got some time delay depends on the Hळ progressive. Finally, a numerical example is given to demonstrate the effectiveness and feasibility of the simulation we give $\mathrm{H} \infty$ controller.
\end{abstract}

\section{Introduction}

In actual industrial control field, there is often some of the nonlinear complex systems, and can not use the precise mathematical model to describe these complex systems, and therefore can not use classical control theory to control for these no precise mathematical models of complex systems . Intelligent control theory in this context is generated, which, TS fuzzy control theory is one of the more extensive use of the theory. Since Takagi-Sugeno in 1985 for the first time since the proposed TS fuzzy model, the theoretical design using TS fuzzy controller nonlinear complex systems, it has already achieved a lot. By TS fuzzy modeling method based on linear control theory can be used in some of the controller design method for control of the complex nonlinear systems. The main features of TS fuzzy system is a nonlinear system via fuzzy sets to be converted into a simple set of subsystems, is complicated by the nonlinear system model "fuzzy" to achieve fuzzy model of the whole system. Many existing research has proven you can take advantage of the linear sequence of TS fuzzy model rules to approximate any smooth nonlinear complex control system. In addition, the use of parallel distributed compensation controller can approximate any smooth nonlinear complex state feedback controller. In this paper, for a class of nonlinear complex systems through a TS fuzzy neural network mathematical model to represent the system, and then use Lyapunov-Krasovskii functional and LMI technique, get the stability conditions of the closed-loop system, and gives $\mathrm{H} \infty$ controller design.

\section{Theoretical Model}

By following our fuzzy neural network model to represent a class of nonlinear complex systems:

$$
\left\{\begin{array}{l}
\ell(t)=-C_{s} x(t)+A_{s} f(x(t))+B_{s} f(x(t-\tau(t)))+D_{s} u(t)+E_{s} \omega(t) \\
y(t)=L_{s} x(t) \\
x(t)=\varphi(t)
\end{array}\right.
$$

Where $\theta(t)=\left[\theta_{1}(t), \theta_{2}(t), \ldots \theta_{n}(t)\right]^{T}$ is the premise variable, $M_{s l}, s \in L=\{1,2, \ldots n\}$ is a fuzzy set, and $\mathrm{m}$ is the number of fuzzy rules. $\mathrm{x}(\mathrm{t})$ is the state vector neural network. $\mathrm{u}(\mathrm{t})$ represents the system control vector; $\mathrm{y}(\mathrm{t})$ denotes a control output vector; $\mathrm{C}=\operatorname{diag}\{\mathrm{C} 1, \mathrm{C} 2, \ldots, \mathrm{Cn}\}$ is the state vector of the 
feedback coefficient. A and B, respectively, represent the neural network link weight matrix and delay link weight matrix. For any $t_{1} \neq t_{2}$, activation function of neural networks to meet:

$$
\alpha_{i} \leq \frac{f_{i}\left(t_{1}\right)-f_{i}\left(t_{2}\right)}{t_{1}-t_{2}} \leq \beta_{i}
$$

TS fuzzy model based on the theory, we can equation (1) is converted to the following model:

$$
\left\{\begin{array}{l}
x(t)=\sum_{s=1}^{m} \frac{\omega_{s}\left[-C_{s} x(t)+A_{s} f(x(t))+B_{s} f(x(t-\tau(t)))+D_{s} u(t)+E_{s} \omega(t)\right]}{\omega_{s}(\theta(t))} \\
y(t)=\sum_{s=1}^{m} \eta_{s}(\theta(t)) L_{s} x(t) \\
x(t)=\varphi(t) \\
t \leq t_{0}
\end{array}\right.
$$

According to decentralized control theory, we use parallel distributed compensation (PDC) method can get the following mathematical model of fuzzy controller. The main features of TS fuzzy system is a nonlinear system via fuzzy sets to be converted into a simple set of subsystems, is complicated by the nonlinear system model "fuzzy" to achieve fuzzy model of the whole system (Fig.1).

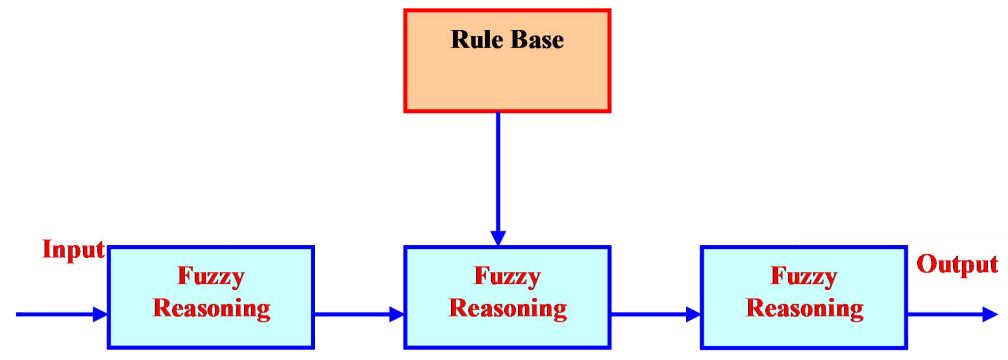

Figure.1 Fuzzy logic diagram

Many references have proved can use a linear sequence of TS fuzzy model rules to approximate any smooth nonlinear complex control system. In addition, the use of parallel distributed compensation controller can approximate any smooth nonlinear complex state feedback controller.

\section{Results of Numerical Simulation}

In the study of control problems, because the reason for the time delays of the control system is always there, so the control system is always behind the action theoretically expected values. When the time accuracy of the control system is designed, when high demands, such a time lag problems will be more prominent. In the design and application of the system, since the limited neuronal conversion rate of the amplifier, the inevitable time lag occurs. Studies have shown that: the time lag always exists for this phenomenon can lead to instability and vibration system, the control system will become more complex, so the stability of variable delay neural networks is very meaningful discussions. In order to carry a proof of our conclusions are correct, we will give an example to show the effectiveness of digital simulation and feasibility of this chapter conclusions. Consider the following uncertain fuzzy time-delay neural network model:

$$
\left\{\begin{array}{l}
\&(t)=-C_{1} x(t)+A_{1} f(x(t))+B_{1} f(x(t-\tau(t)))+D_{1} u(t)+E_{1} \omega(t) \\
y(t)=L_{1} x(t)
\end{array}\right.
$$

After calculation we can know: 


$$
1 \leq \tau(t) \leq 1.5, \text {, d }(t) \leq 0.5, F_{1}=\left[\begin{array}{cc}
-1 & 0 \\
0 & -1
\end{array}\right]
$$

and $F_{2}=\left[\begin{array}{ll}0 & 0 \\ 0 & 0\end{array}\right]$

Equation can be solved through the use of Matlab LMI in Control Toolbox (1) of the LMI, the LMI toolbox of Matlab simulation system. Fig. 2 shows the closed-loop system to control the output at outside interference. From the Matlab simulation results can be concluded that the role of the system due to the control that the closed loop system satisfies $\mathrm{H} \infty$ asymptotically stable.

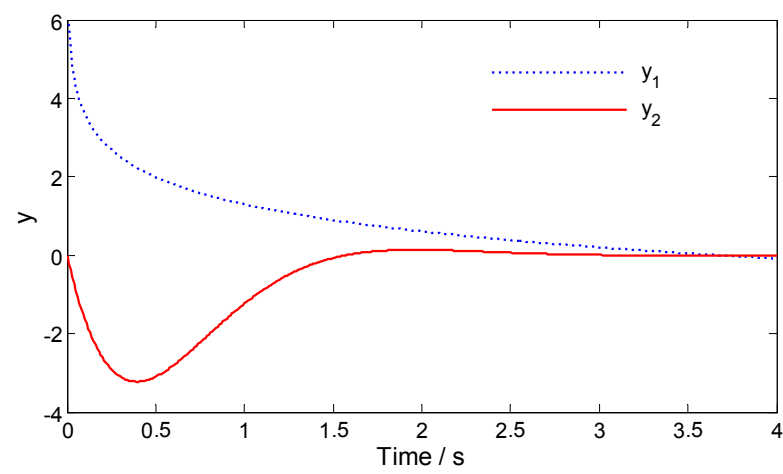

Figure.2 Our model outside interference closed-loop system output

In the past few decades, due to the neural network classification model, associative memory, image processing, quadratic optimization, and applications in other fields have been experts in many areas of attention and concern, and have made many important research results. In addition, since 1985 the concept of TS fuzzy model was first proposed, the model has attracted a growing number of scientists in various fields of concern and interest, and in the past few decades, the stability analysis of fuzzy systems has achieved fruitful results(Fig.3).

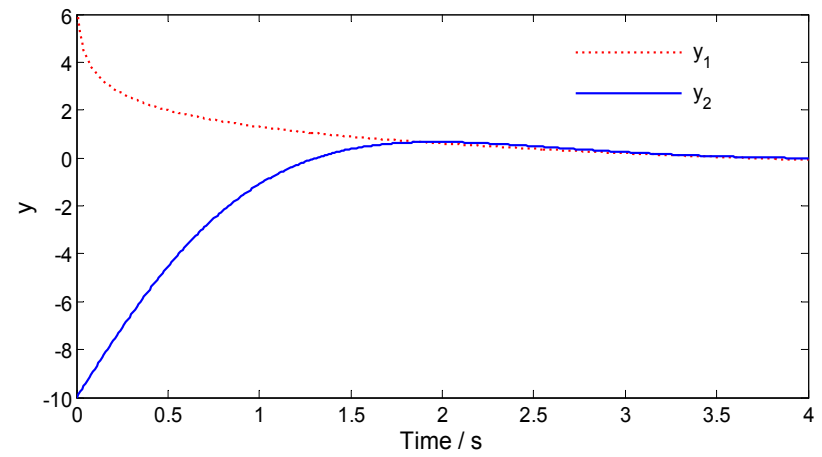

Figure.3 TS model outside interference closed-loop system output

With the deepening of neural networks, fuzzy neural network mathematical model, time delay neural networks, neural networks, and with a pulse reaction-diffusion neural network has been more and more attention. The main work of this paper is based on the literature about the stability analysis of neural networks already on; continue to discuss the stability with time varying delay fuzzy neural network, fuzzy neural pulses with time-varying delays and reaction-diffusion stability and stability of the network with time-varying delay discrete fuzzy pulse neural network.

\section{Conclusion}

Research in the field of neural networks has made significant progress, that progresses has attracted a lot of attention and support of the people more money on. Now more and more academic and commercial research is carried out on neural networks, such as chip-based neural networks are developed and applied, and produces a number of complex issues, and treats these problems solved. Obviously, now just a transition period of neural networks. Neural network results from the ability of complex data extraction can be used to extract or detect those patterns for humans or other computer 
technology is too complex hard to notice a trend. A class of design problems $\mathrm{H} \infty$ fuzzy neural network controller. By using Lyapunov-Krasovskii functional theory and judgment theorem to derive a stable process introduces several additional matrices, got some time delay depends on the Hळ progressive. Finally, a numerical example is given to demonstrate the effectiveness and feasibility of the simulation we give $\mathrm{H} \infty$ controller.

\section{Acknowledgment}

The authors thank the funding from Department of Science and Technology Foundation in Jilin Province (20140101198JC).

\section{References}

[1] M. Young, The Technical Writer's Handbook. Mill Valley, CA: University Science, 1989.

[2] G.S. Ng, C. Quek, H. Jiang: FCMAC-EWS: A bank failure early warning system based on a novel localized pattern learning and semantically associative fuzzy neural network, Expert Systems with Applications, Vol.34 (2008), p.989-1003.

[3] R. R. Yager: Implementing fuzzy logic controllers using a neural network framework, Fuzzy Sets and Systems, Vol.48 (1992), p.53-64.

[4] W.L. Tung, C. Quek: Financial volatility trading using a self-organising neural-fuzzy semantic network and option straddle-based approach, Expert Systems with Applications, Vol.38 (2011), p.4668-4688.

[5] N. Kasabov, K. Dhoble, N. Nuntalid, G. Indiveri: Dynamic evolving spiking neural networks for on-line spatio- and spectro-temporal pattern recognition, Neural Networks, Vol.41 (2013), p.188-201.

[6] Y. Chong, C. Quek, P. Loh: A novel neuro-cognitive approach to modeling traffic control and flow based on fuzzy neural techniques, Expert Systems with Applications, Vol.36 (2009), p.4788-4803.

[7] W. Pedrycz: Fuzzy neural networks and neurocomputations, Fuzzy Sets and Systems, Vol.56 (1993), p.1-28.

[8] F. Gaxiola, P. Melin, F. Valdez, O. Castillo: Interval type-2 fuzzy weight adjustment for backpropagation neural networks with application in time series prediction, Information Sciences, Vol.260 (2014), p.1-14.

[9] R. Eslamloueyan: Designing a hierarchical neural network based on fuzzy clustering for fault diagnosis of the Tennessee-Eastman process, Applied Soft Computing, Vol.11 (2011), p.1407-1415.

[10]R. A. Aliev, B.G. Guirimov, Bijan Fazlollahi, R.R. Aliev: Evolutionary algorithm-based learning of fuzzy neural networks. Part 2: Recurrent fuzzy neural networks, Fuzzy Sets and Systems, Vol.160 (2009), p.2553-2566. 